PROCEEDINGS OF THE

AMERICAN MATHEMATICAL SOCIETY

Volume 132, Number 7 , Pages 1979-1985

S 0002-9939(03)07321-0

Article electronically published on December 15, 2003

\title{
ZERO PRODUCT PRESERVING MAPS OF OPERATOR-VALUED FUNCTIONS
}

\author{
WEN-FONG KE, BING-REN LI, AND NGAI-CHING WONG
}

(Communicated by David R. Larson)

\begin{abstract}
Let $X, Y$ be locally compact Hausdorff spaces and $\mathcal{M}, \mathcal{N}$ be Banach algebras. Let $\theta: C_{0}(X, \mathcal{M}) \rightarrow C_{0}(Y, \mathcal{N})$ be a zero product preserving bounded linear map with dense range. We show that $\theta$ is given by a continuous field of algebra homomorphisms from $\mathcal{M}$ into $\mathcal{N}$ if $\mathcal{N}$ is irreducible. As corollaries, such a surjective $\theta$ arises from an algebra homomorphism, provided that $\mathcal{M}$ is a $W^{*}$-algebra and $\mathcal{N}$ is a semi-simple Banach algebra, or both $\mathcal{M}$ and $\mathcal{N}$ are $C^{*}$-algebras.
\end{abstract}

\section{INTRODUCTION}

We are interested in the question how the zero product structure of a Banach algebra determines its full algebraic structure. For example, two abelian $C^{*}$-algebras are *-isomorphic if there exists a bijective linear map between them preserving zero products (13, 11, 14]). On the other hand, bounded bijective linear zero product preservers of nonabelian $C^{*}$-algebras also provide algebraic ${ }^{*}$-isomorphisms [7]. Recently, Araujo and Jarosz [2] showed that the existence of a bijective linear map of standard operator algebras preserving zero products in both ways also implies that they are isomorphic as Banach algebras. In this paper, we study bounded zero product preservers between Banach algebras of operator-valued functions. We show that such maps are given by continuous fields of algebra homomorphisms in many situations.

Let $X$ be a locally compact Hausdorff space. Denote by $X_{\infty}=X \cup\{\infty\}$ the onepoint compactification of $X$. In case $X$ is already compact, $\infty$ is an isolated point in $X_{\infty}$. For a real or complex Banach algebra $\mathcal{M}$, let $C_{0}(X, \mathcal{M})=\{f \in C(X, \mathcal{M})$ : $f(\infty)=0\}$ be the Banach algebra of all continuous vector-valued functions from $X$ into $\mathcal{M}$ vanishing at infinity. Note that $C_{0}(X, \mathcal{M})$ is isometrically and algebraically isomorphic to the (projective) tensor product $C_{0}(X) \otimes \mathcal{M}$.

In this paper, we shall study those bounded linear maps $\theta$ from $C_{0}(X, \mathcal{M})$ into another such algebra $C_{0}(Y, \mathcal{N})$ preserving zero products. Namely, $f g=0$ implies $\theta(f) \theta(g)=0$. In other words,

$$
f(x) g(x)=0 \text { in } \mathcal{M} \text { for all } x \in X \Longrightarrow \theta(f)(y) \theta(g)(y)=0 \text { in } \mathcal{N} \text { for all } y \in Y .
$$

\footnotetext{
Received by the editors July 25, 2002 and, in revised form, March 7, 2003.

2000 Mathematics Subject Classification. Primary 46E40, 47B33.

Key words and phrases. Zero product preserving maps, Banach algebra homomorphisms.
} 
For example, let $\sigma: Y \rightarrow X$ be a continuous function, let $h$ be a uniformly bounded norm continuous function from $Y$ into the center of $\mathcal{N}$, and let $\varphi$ be a uniformly bounded SOT continuous function from $Y$ into $B(\mathcal{M}, \mathcal{N})$ such that each $\varphi_{y}=\varphi(y)$ is an algebra homomorphism. Then

$$
\theta(f)(y)=h(y) \varphi_{y}(f(\sigma(y)))
$$

defines a zero product preserving bounded linear map from $C_{0}(X, \mathcal{M})$ into $C_{0}(Y, \mathcal{N})$. In particular, $\theta=h \varphi$ for a bounded central element $h$ in the algebra $C(Y, \mathcal{N})$ and an algebra homomorphism $\varphi$ from $C_{0}(X, \mathcal{M})$ into $C_{0}(Y, \mathcal{N})$. We will investigate when zero product preserving bounded linear maps arise in this way.

For the scalar case, every zero product preserving bounded linear map $\theta$ from $C_{0}(X)$ into $C_{0}(Y)$ is of the expected form (1.1) (13], [11, [14]). Recall that a subalgebra $\mathcal{S}$ of the algebra $B(E)$ of all bounded linear operators on a Banach space $E$ is said to be standard if $\mathcal{S}$ contains the identity operator and all continuous finite rank operators. Using an interesting geometric approach, Araujo and Jarosz [2] showed that when $X, Y$ are real compact and $\mathcal{M}$ and $\mathcal{N}$ are standard operator algebras, every bijective linear map from $C(X, \mathcal{M})$ onto $C(Y, \mathcal{N})$ preserving zero products in both directions is in the form of (1.1). However, in the non-bijective case, it becomes a very difficult task without assuming continuity. Even discontinuous algebra homomorphisms have complicated structure ([15], 19]). Finally, readers are referred to [1, 12], [6], and [21] for problems of similar interests.

\section{Results}

A linear map $\theta$ from $C_{0}(X, \mathcal{M})$ into $C_{0}(Y, \mathcal{N})$ is said to be strictly separating if

$$
\|f(x)\|\|g(x)\|=0 \text { for all } x \in X \quad \Longrightarrow \quad\|\theta f(y)\|\|\theta g(y)\|=0 \text { for all } y \in Y \text {. }
$$

Denote by $\operatorname{coz}(f)=\{x \in X: f(x) \neq 0\}$ the cozero set of an $f$ in $C_{0}(X, \mathcal{M})$. Then $\theta$ is strictly separating if and only if it preserves the disjointness of cozeroes. We note that a subset $U$ of $X$ is the cozero of a continuous function in $C_{0}(X, \mathcal{M})$ if and only if $U$ is $\sigma$-compact and open. For any $\sigma$-compact open subset $U$ of $X$, denote by $C_{0}(U, \mathcal{M})$ the subalgebra of all $f$ in $C_{0}(X, \mathcal{M})$ with $\operatorname{coz}(f) \subseteq U$.

A Banach algebra $\mathcal{N}$ is said to be irreducible if it has a faithful irreducible representation $\pi: \mathcal{N} \rightarrow B(E)$, where $E$ is a Banach space. Recall that every irreducible representation of a Banach algebra is automatically bounded [15].

Theorem 1. Let $X$ and $Y$ be locally compact Hausdorff spaces. Let $\mathcal{M}$ and $\mathcal{N}$ be Banach algebras such that $\mathcal{N}$ is irreducible, and let $\theta$ be a continuous zero product preserving linear map from $C_{0}(X, \mathcal{M})$ into $C_{0}(Y, \mathcal{N})$ with dense range. Then $\theta$ is strictly separating.

Indeed, there exists a continuous map $\sigma: Y \rightarrow X$, and for each $y$ in $Y$ a bounded zero product preserving linear map $H_{y}: \mathcal{M} \rightarrow \mathcal{N}$ with dense range such that

$$
\theta(f)(y)=H_{y}(f(\sigma(y))) \quad \text { for all } f \in C_{0}(X, \mathcal{M}) \text { and } y \in Y .
$$

Moreover, the correspondence $y \mapsto H_{y}$ defines a uniformly bounded map $H: Y \rightarrow$ $B(\mathcal{M}, \mathcal{N})$ continuous in the strong operator topology (SOT).

Proof. Let $\pi: \mathcal{N} \rightarrow B(E)$ be a faithful irreducible representation of $\mathcal{N}$. Composing $\theta$ with $\pi$, we can assume that $\mathcal{N}$ is an irreducible subalgebra of $B(E)$ and that $\theta$ is again bounded and zero product preserving with dense range. 
Fix $y$ in $Y$, and denote by

$$
\begin{array}{r}
S_{y}=\left\{x \in X_{\infty}: \text { for each } \sigma \text {-compact open neighborhood } U \text { of } x,\right. \\
\text { there is an } \left.f \text { in } C_{0}(U, \mathcal{M}) \text { such that } \theta(f)(y) \neq 0\right\} .
\end{array}
$$

Claim 1. $S_{y} \neq \varnothing$.

Suppose not, and for each $x$ in $X_{\infty}$, suppose there is a $\sigma$-compact open neighborhood $U$ of $x$ such that $\left.\theta\right|_{C_{0}(U, \mathcal{M})}$ is trivial at $y$. Write

$$
X_{\infty}=U_{0} \cup U_{1} \cup \cdots \cup U_{n}
$$

for $x_{0}=\infty$, and some $x_{1}, \ldots, x_{n}$ in $X$, with a $\sigma$-compact open neighborhood $U_{i}$ for $i=0,1, \ldots, n$, respectively. Let

$$
1=f_{0}+f_{1}+\cdots+f_{n}
$$

be a continuous partition of unity such that $\operatorname{coz} f_{i} \subseteq U_{i}$ for $i=0,1, \ldots, n$. Then for all $f$ in $C_{0}(X, \mathcal{M})$,

$$
\theta(f)(y)=\theta\left(f_{0} f+f_{1} f+\cdots+f_{n} f\right)(y)=0,
$$

since $\operatorname{coz}\left(f_{i} f\right) \subseteq U_{i}$ for each $i=0,1, \ldots, n$. This is impossible.

Claim 2. $x_{1}, x_{2} \in S_{y} \Longrightarrow x_{1}=x_{2}$.

Suppose $x_{2} \neq x_{1}$ and $x_{1} \neq \infty$. Let $U_{1}$ and $U_{2}$ be disjoint $\sigma$-compact open neighborhoods of $x_{1}$ and $x_{2}$, respectively. We can assume that $\infty \notin U_{1}$. Since

$$
f_{1} f_{2}=f_{2} f_{1}=0 \quad \text { for all } f_{i} \in C_{0}\left(U_{i}, \mathcal{M}\right), \quad i=1,2,
$$

we have

$$
\theta\left(f_{1}\right) \theta\left(f_{2}\right)=\theta\left(f_{2}\right) \theta\left(f_{1}\right)=0 \quad \text { in } C_{0}(Y, \mathcal{N}) .
$$

Let $E_{1}$ be the intersection of the kernels of all $\theta\left(f_{1}\right)(y)$ with $f_{1}$ in $C_{0}\left(U_{1}, \mathcal{M}\right)$. Because both $\left.\theta\right|_{C_{0}\left(U_{1}, \mathcal{M}\right)}$ and $\left.\theta\right|_{C_{0}\left(U_{2}, \mathcal{M}\right)}$ are not trivial at $y$, we have that $E_{1}$ is a proper nontrivial subspace of $E$, that is, $\{0\} \neq E_{1} \neq E$.

Let $V$ be a nonempty open set in $Y$ such that $\bar{V} \subseteq U_{1}$. Let $g$ be in $C_{0}(X)$ such that $\operatorname{coz} g \subseteq U_{1}$ and $\left.g\right|_{V}=1$. For each $f$ in $C_{0}(X, \mathcal{M})$, write

$$
f=f g+f(1-g) \text {. }
$$

Since $\operatorname{coz}(f g) \subseteq U_{1}$, we have

$$
\left.\theta(f g)(y)\right|_{E_{1}}=0 .
$$

Hence

$$
\left.\theta(f)(y)\right|_{E_{1}}=\left.\theta(f(1-g))(y)\right|_{E_{1}} .
$$

For any $k$ in $C_{0}(X, \mathcal{M})$ with coz $k \subseteq V$, we have $k(f(1-g))=0$. This implies

$$
\left.\theta(k)(y) \theta(f)(y)\right|_{E_{1}}=\left.\theta(k)(y) \theta(f(1-g))(y)\right|_{E_{1}}=0 \quad \text { for all } f \in C_{0}(X, \mathcal{M}) .
$$

However, $\left\{\theta(f)(y): f \in C_{0}(X, \mathcal{M})\right\}$ is dense in $\mathcal{N}$, which is irreducible on $E$. Therefore,

$$
\theta(k)(y)=0 \quad \text { for all } k \in C_{0}(X, \mathcal{M}) \text { with } \operatorname{coz} k \subseteq V .
$$

Since $V$ is an arbitrary nonempty open set with closure contained in $U_{1}$, we have

$$
\theta(k)(y)=0 \quad \text { for all } k \in C_{0}\left(U_{1}, \mathcal{M}\right) .
$$

This conflict establishes Claim 2.

By Claims 1 and 2, $S_{y}$ is a singleton. 
Claim 3. If $S_{y}=\{x\}$, then

$$
f(x)=0 \quad \Longrightarrow \quad \theta(f)(y)=0 .
$$

By Urysohn's Lemma, we can assume that $f$ vanishes in a neighborhood of $x$. Now $x \notin \overline{\operatorname{coz} f}$, which is compact in $X_{\infty}$. For each $x^{\prime}$ in $\overline{\operatorname{coz} f}$, there is a $\sigma$-compact open neighborhood $U^{\prime}$ of $x^{\prime}$ such that $\left.\theta\right|_{C_{0}\left(U^{\prime}, \mathcal{M}\right)}$ is trivial at $y$. By a compactness argument similar to the one proving Claim 1, we see that $\theta(f)(y)=0$.

It follows from Claim 3 that $S_{y} \neq\{\infty\}$ for all $y$ in $Y$ since $\theta$ has dense range. Denote by $\sigma(y)=x$ if $S_{y}=\{x\}$. Then there is a linear map $H_{y}: \mathcal{M} \rightarrow \mathcal{N}$ such that

$$
\theta(f)(y)=H_{y}(f(\sigma(y))) \quad \text { for all } f \in C_{0}(X, \mathcal{M}) \text { and } y \in Y .
$$

In particular, $\theta$ is strictly separating.

The rest of the proof follows in a straightforward manner, or one can quote the standard results about strictly separating maps in [6], [12].

The following lemma might be known, although we do not find a proof from the literature. Remark that it is shown in [17] that every nonzero Banach algebra homomorphism from $B(H)$ into $B(K)$ is injective if both $H$ and $K$ are separable Hilbert spaces. However, there is an example in [18] of a nonzero homomorphism from $B(H)$ into $B(H)$ with compact operators as its kernel, where $H$ is inseparable. Moreover, it is known that every irreducible representation of a Banach algebra is norm continuous [15] and every algebra isomorphism between $C^{*}$-algebras is a *isomorphism [20 Theorem 4.1.20].

Lemma 2. Let $H, K$ be real or complex Hilbert spaces of arbitrary dimension. Let $B(H)$ and $B(K)$ be the algebras of all bounded linear operators on $H$ and $K$, respectively. Then every surjective algebra homomorphism from $B(H)$ onto $B(K)$ is an isomorphism.

Proof. The case is trivial when $H$ is of finite dimension since $B(H)$ is then a simple algebra. Suppose the (Hilbert space) dimension of $H$ is an infinite cardinal number $\aleph_{H}$. For each infinite cardinal number $\aleph \leq \aleph_{H}$, let $I_{\aleph}$ be the closed two-sided ideal of $B(H)$ consisting of operators $T$ such that all closed subspaces contained in the range of $T$ are of dimension less than $\aleph$. In case $H$ is separable, $I_{\aleph_{H}}=\mathcal{K}(H)$, the ideal of compact operators on $H$. In general, as indicated in [5], $I_{\aleph_{H}}$ is the largest two-sided ideal of $B(H)$. In fact, every closed two-sided ideal of $B(H)$ is in the form of $I_{\aleph}$ for some $\aleph \leq \aleph_{H}$ [9, Section 17].

Let $\theta$ be an algebra homomorphism from $B(H)$ onto $B(K)$. Then the kernel $I$ of $\theta$ is a closed two-sided ideal of $B(H)$. Since the quotient algebra $B(H) / I$ is isomorphic to $B(K)$, there is an $e$ in $B(H)$ such that $(e+I) B(H)(e+I)=e B(H) e+I$ is of dimension one modulo $I$. Assume $I$ is nonzero. Let $\aleph$ be the infinite cardinal number such that $I=I_{\aleph}$. Then the range of $e$ contains a closed subspace of dimension $\aleph$. By halving this subspace into two, each of dimension $\aleph$, we see that $e B(H) e$ contains two elements linearly independent modulo $I_{\aleph}$, a contradiction. This completes our proof.

Corollary 3. Let $X, Y$ be locally compact Hausdorff spaces. Let $\mathcal{M}, \mathcal{N}$ be either the Banach algebras $B(H), B(K)$ of all bounded operators or the algebras $\mathcal{K}(H), \mathcal{K}(K)$ of compact operators on real or complex Hilbert spaces $H, K$, respectively. Let $\theta: C_{0}(X, \mathcal{M}) \rightarrow C_{0}(Y, \mathcal{N})$ be a continuous surjective zero product preserving linear map. Then there exist a continuous function $\sigma$ from $Y$ into $X$, a continuous scalar 
function $h$ on $Y$, and a SOT continuous map $y \mapsto S_{y}$ from $Y$ into $B(K, H)$ such that $S_{y}$ is invertible and

$$
\theta(f)(y)=h(y) S_{y}^{-1} f(\sigma(y)) S_{y}, \quad \forall f \in C(X, \mathcal{M}), \forall y \in Y .
$$

Proof. It follows from Theorem 1 that for each fixed $y$ in $Y, \theta$ induces a bounded zero product preserving linear map $H(y)$ from $\mathcal{M}$ onto $\mathcal{N}$. By either [10, Theorem 2.1] or [7, Corollary 3.2], $H(y)$ is a scalar multiple of a bounded algebra homomorphism from $\mathcal{M}$ onto $\mathcal{N}$. Since $\mathcal{K}(H)$ is simple, this algebra homomorphism is indeed an isomorphism if $\mathcal{M}$ and $\mathcal{N}$ are $\mathcal{K}(H)$ and $\mathcal{K}(K)$, respectively. On the other hand, by Lemma 2 the algebra homomorphism above is again an isomorphism in case $\mathcal{M}$ and $\mathcal{N}$ are $B(H)$ and $B(K)$, respectively. Thus, by either [3, Theorem 4] or [8. Corollary 3.2], there exist a scalar $h(y)$ and a bounded invertible operator $S_{y}$ on $K$ to implement (2.1). It is then routine to check the continuity of $h$ and the map $y \mapsto S_{y}$.

The following corollary holds, for example, when $\mathcal{M}$ is a $W^{*}$-algebra, or a unital $C^{*}$-algebra of real rank zero 4 .

Corollary 4. Let $X$ and $Y$ be locally compact Hausdorff spaces such that $X$ is compact. Let $\mathcal{M}$ be a unital Banach algebra such that the subalgebra of $\mathcal{M}$ generated by its idempotents is norm dense in $\mathcal{M}$, and let $\mathcal{N}$ be a semi-simple Banach algebra. Let $\theta$ be a continuous zero product preserving linear map from $C(X, \mathcal{M})$ into $C_{0}(Y, \mathcal{N})$ with dense range. Then $\theta(1)$ is in the center of $C_{0}(Y, \mathcal{N})$, and

$$
\theta(1) \theta(f g)=\theta(f) \theta(g) \quad \text { for all } f, g \in C(X, \mathcal{M}) \text {. }
$$

Suppose, in addition, that $Y$ is compact and $\mathcal{N}$ is unital. If $\theta(1)$ is invertible or $\theta$ is surjective, then $\theta=\theta(1) \varphi$ for an algebra homomorphism $\varphi$.

Proof. Let $\pi: \mathcal{N} \rightarrow B(E)$ be an irreducible representation of $\mathcal{N}$. Then $\theta_{\pi}=$ $\pi \circ \theta$ is again a continuous zero product preserving linear map from $C(X, \mathcal{M})$ into $C_{0}(Y, \pi(\mathcal{N}))$ with dense range. By Theorem 1, we find that $\theta_{\pi}$ carries a weighted composition operator form

$$
\theta_{\pi}(f)(y)=H_{y}(f(\sigma(y))) \quad \text { for all } f \in C(X, \mathcal{M}) \text { and } y \in Y .
$$

In particular, each $H_{y}$ is a continuous zero product preserving linear map from $\mathcal{M}$ into $\pi(\mathcal{N})$ with dense range.

By results in [10] (see also [7]), for each $y$ in $Y$ we have that $\theta_{\pi}(1)(y)=H_{y}(1)$ is in the center of $\mathcal{N}$ and

$$
H_{y}(1) H_{y}(a b)=H_{y}(a) H_{y}(b) \text { for all } a, b \in \mathcal{M} .
$$

Hence

and

$$
\pi(\theta(1) \theta(f)-\theta(f) \theta(1))=0
$$

$$
\pi(\theta(1) \theta(f g)-\theta(f) \theta(g))=0
$$

for all $f, g$ in $C(X, \mathcal{M})$. Being semi-simple, $\mathcal{N}$ has a faithful family of irreducible representations. Thus $\theta(1)$ is in the center of $C_{0}(Y, \mathcal{N})$ and (2.2) holds.

Now, we assume that $Y$ is compact and $\mathcal{N}$ is unital. If $\theta$ is surjective, $1=\theta(f)$ for some $f$ in $C(X, \mathcal{M})$. It follows from $\theta(1) \theta\left(f^{2}\right)=\theta(f)^{2}=1$ that $\theta(1)$ is invertible. Assume $\theta(1)$ is invertible. Then $\theta(1)^{-1} \theta$ is again a bounded zero product preserving linear map with dense range, and sends 1 to 1 . Suppose now that $\theta(1)=1$. Then (2.2) ensures that $\theta$ is an algebra homomorphism. 
A recent result in [7] states that every surjective zero product preserving bounded linear map $\theta$ between unital $C^{*}$-algebras is a product $\theta=\theta(1) \varphi$ of the invertible central element $\theta(1)$ and an algebra homomorphism $\varphi$. Since $C(X, \mathcal{A})($ resp. $C(Y, \mathcal{B}))$ is $*$-isomorphic to the (projective) tensor product $C(X) \otimes \mathcal{A}$ (resp. $C(Y) \otimes \mathcal{B})$ as $C^{*}$-algebras (see, e.g., [16]), we have the following.

Corollary 5. Let $X$ and $Y$ be compact Hausdorff spaces, and $\mathcal{A}, \mathcal{B}$ be unital $C^{*}$ algebras. Let $\theta$ be a continuous zero product preserving linear map from $C(X, \mathcal{A})$ onto $C(Y, \mathcal{B})$. Then $\theta(1)$ is an invertible element in the center of $C(Y, \mathcal{B})$, and $\theta=\theta(1) \varphi$ for an algebra homomorphism $\varphi$.

The following example shows that the irreducibility condition on $\mathcal{N}$ cannot be dropped in Theorem 1 and the map $\theta$ in the Corollaries 4 and 5 cannot be written as a weighted composition operator in the form of (1.1) in general.

Example 6. Let $X=\{0\}$ and $\mathcal{M}=\mathbb{C} \oplus \mathbb{C}$ be the two-dimensional $C^{*}$-algebra, and let $Y=\{1,2\}$ and $\mathcal{N}=\mathbb{C}$ be the one-dimensional $C^{*}$-algebra. Define $\theta$ : $C(X, \mathcal{M}) \rightarrow C(Y, \mathcal{N})$ by $\theta(a \oplus b)=g$ with $g(1)=a$ and $g(2)=b$. Then $\theta$ is bijective and preserves zero products in both directions.

Remark that $\theta: C(X, \mathcal{M}) \rightarrow C(Y, \mathcal{N})$ satisfies the condition stated in Theorem 1 In fact, let $h_{1}(a \oplus b)=a$ and $h_{2}(a \oplus b)=b$ be the canonical projection of $\mathbb{C} \oplus \mathbb{C}$ onto its summands, and set $\sigma(1)=\sigma(2)=0$. Then

$$
\theta(f)(y)=h_{y}(f(\sigma(y))), \quad \forall f \in C(X, \mathcal{M}), \forall y \in Y .
$$

However, $\mathcal{M}$ is not irreducible and $T^{-1}: C(Y, \mathcal{N}) \rightarrow C(X, \mathcal{M})$ does not carry a weighted composition operator form. Note also that $X$ and $Y$ are not homeomorphic although both $C(X, \mathcal{M})$ and $C(Y, \mathcal{N})$ are isomorphic to $\mathbb{C} \oplus \mathbb{C}$ as $C^{*}$-algebras and $\theta$ implements an algebra isomorphism between them.

\section{ACKNOWLEDGMENTS}

We would like to express our gratitude to Matej Brešar, Mikhail A. Chebotar, Pjek-Hwee Lee and Peter Šemrl for many helpful discussions. Thanks are also due to the referee for some useful advice.

\section{REFERENCES}

[1] Y. A. Abramovich, Multiplicative representation of disjointness preserving operators, Nederl. Akad. Wetensch. Indag. Math. 45 (1983), 265-279. MR 85f:47040

[2] J. Araujo and K. Jarosz, Biseparating maps between operator algebras, J. Math. Anal. Appl. 282 (2003), no. 1, 48-55.

[3] B. H. Arnold, Rings of operators on vector spaces, Ann. of Math. (2) 45 (1944), 24-49. MR 5:147c

[4] L. G. Brown and G. K. Pedersen, $C^{*}$-algebras of real rank zero, J. Funct. Anal. 99 (1991), 131-149. MR 92m:46086

[5] J. W. Calkin, Two-sided ideals and congruences in the ring of bounded operators in Hilbert space, Ann. of Math. 42 (1941), 839-873. MR 3:208c

[6] J. T. Chan, Operators with the disjoint support property, J. Operator Theory 24 (1990), 383-391. MR 93c:47035

[7] M. A. Chebotar, W.-F. Ke, P.-H. Lee and N.-C. Wong, Mappings preserving zero products, Studia Math. 155, no. 1 (2003), 77-94. MR 2003m:47066

[8] P. R. Chernoff, Representations, automorphisms, and derivations of some operator algebras, J. Funct. Anal. 12 (1973), 275-289. MR 50:2934

[9] J. B. Conway, A course in operator theory, Graduate Studies in Math. 21, American Mathematical Society, Providence, Rhode Island, 2000. MR 2001d:47001 
[10] J. Cui and J. Hou, Linear maps on von Neumann algebras preserving zero products or TRrank, Bull. Austral. Math. Soc. 65 (2002), 79-91. MR 2002m:46092

[11] J. J. Font and S. Hernández, On separating maps between locally compact spaces, Arch. Math. (Basel) 63 (1994), 158-165. MR 95k:46083

[12] J. E. Jamison and M. Rajagopalan, Weighted composition operator on $C(X, E)$, J. Operator Theory 19 (1988), 307-317. MR 90b:47052

[13] K. Jarosz, Automatic continuity of separating linear isomorphisms, Canad. Math. Bull. 33 (1990), 139-144. MR 92j:46049

[14] J.-S. Jeang and N.-C. Wong, Weighted composition operators of $C_{0}(X)$ 's, J. Math. Anal. Appl. 201 (1996), 981-993. MR 97f:47029

[15] B. E. Johnson, Continuity of homomorphisms of algebras of operators, J. London Math. Soc. 42 (1967), 537-541. MR 35:5953

[16] B.-R. Li, Introduction to operator algebras, World Scientific, Singapore, 1992.

[17] H. Porta and J. T. Schwartz, Representations of the algebra of all operators in Hilbert space, and related analytic function algebras, Comm. Pure and Applied Math. 20 (1967), 457-492. MR 35:2157

[18] H. Porta, A note on homomorphisms of operator algebras, Colloq. Math. 20 (1969), 117-119. MR 39:1988

[19] V. Runde, The structure of discontinuous homomorphisms from non-commutative $C^{*}$ algebras, Glasgow Math. J. 36 (1994), 209-218. MR 95i:46091

[20] S. Sakai, $C^{*}$-algebras and $W^{*}$-algebras, Ergebnisse der Mathematik und ihrer Grenzgebiete, Band 60, Springer-Verlag, New York, 1971. MR 56:1082

[21] M. Wolff, Disjointness preserving operators on $C^{*}$-algebras, Arch. Math. (Basel) 62 (1994), 248-253. MR 94k:46122

Department of Mathematics, National Cheng Kung University, Tainan 701, Taiwan E-mail address: wfke@mail.ncku.edu.tw

Institute of Mathematics, Chinese Academy of Sciences, Beijing 100080, China

E-mail address: brli@mail2.math.ac.cn

Department of Applied Mathematics, National Sun Yat-Sen University, Kaohsiung 804, TAIWAN

E-mail address: wong@math.nsysu.edu.tw 